\title{
Continuous erector spinae plane (ESP) block in breast cancer surgery
}

Monica Pérez-Poquet and Pamela Maria Celdrán-Clusella. Department of Anesthesiology, Hospital Universitari MútuaTerrassa, Terrassa, Spain.

\section{Background and aims}

Erector spinae plane block has recently been reported for thoracic wall procedures. We describe three cases of continuous ESP block in breast cancer surgery.

\section{Case reports}

Three women $(37,42$, and 47 years-old) underwent breast cancer surgery:

- Ist patient: Mastectomy and sentinel lymph node dissection

- 2nd patient: Mastectomy and lymphadenectomy

- 3rd patient: Tumorectomy and lymphadenectomy

ESP block was performed at T2 level with ropivacaine $0.35 \%(30 \mathrm{~mL})$ plus catheter insertion (Fig I and 2).

General anesthesia was maintained with propofol $(7 \mathrm{mg} / \mathrm{Kg} / \mathrm{h})$ and low-dose remifentanil $(0.05 \mathrm{mcg} / \mathrm{Kg} / \mathrm{minute})$. A single dose of fentanyl $(2 \mathrm{mcg} / \mathrm{Kg})$ was used in the induction. All patients received intraoperative antiemetic prophylaxis, dexketoprofen and paracetamol.

Postoperative pain control: Continuous ESP block with ropivacaine $0.2 \%$ infusion $(10 \mathrm{~mL} / \mathrm{h})$, paracetamol and dexketoprofen. Pain was measured with VAS score (0-10) when patients arrived at the post-anesthesia care unit (PACU), when they left PACU and 24 hours after surgery.

Results: Patients reported VAS of $0-\mathrm{I}$ at all endpoints without requiring any additional analgesia. After ESP catheter infusion was stopped the analgesia lasted 6-8 hours.

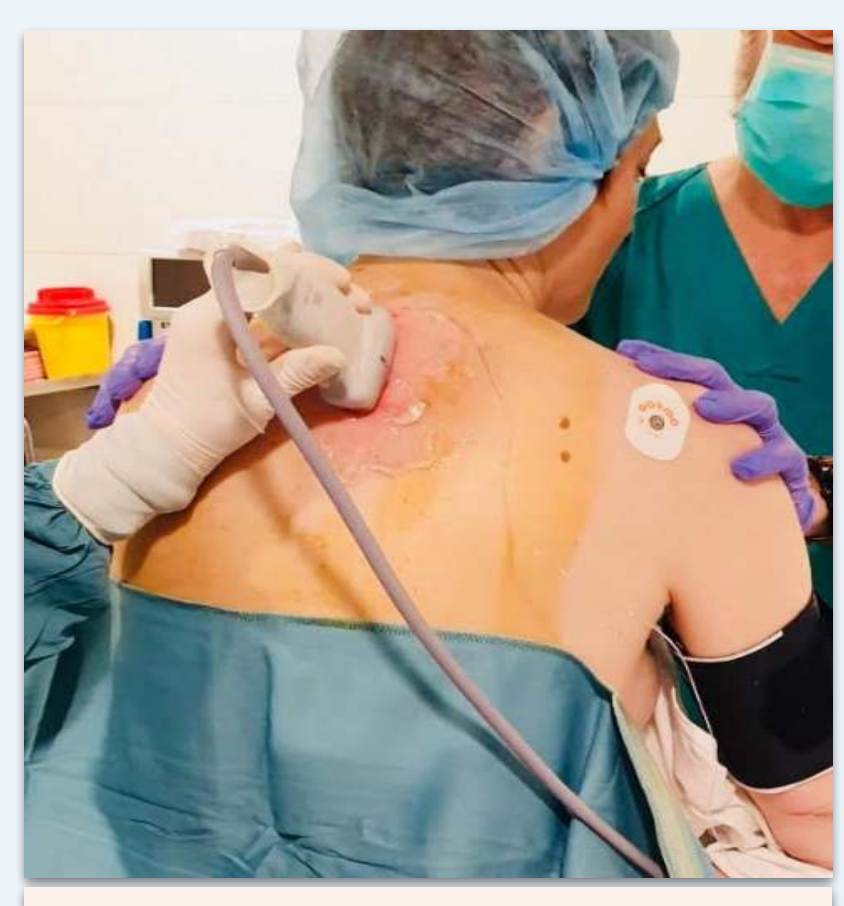

Fig I. ESP block: US probe placement

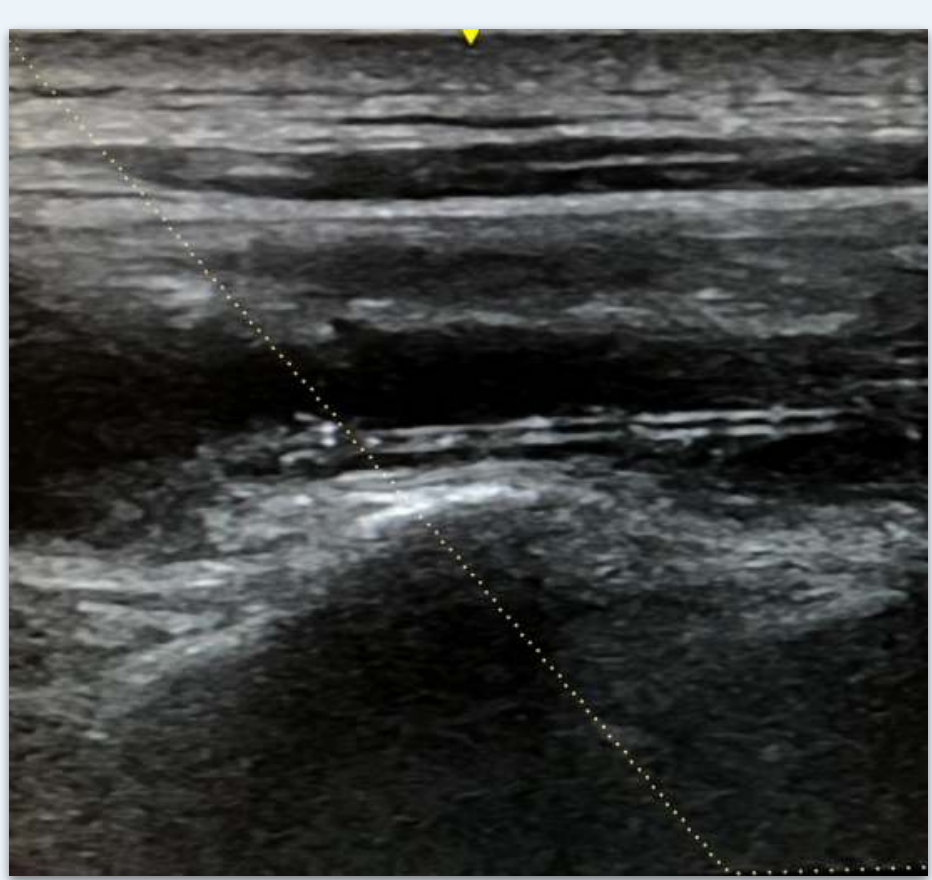

Fig 2. ESP block: US image LA and catheter

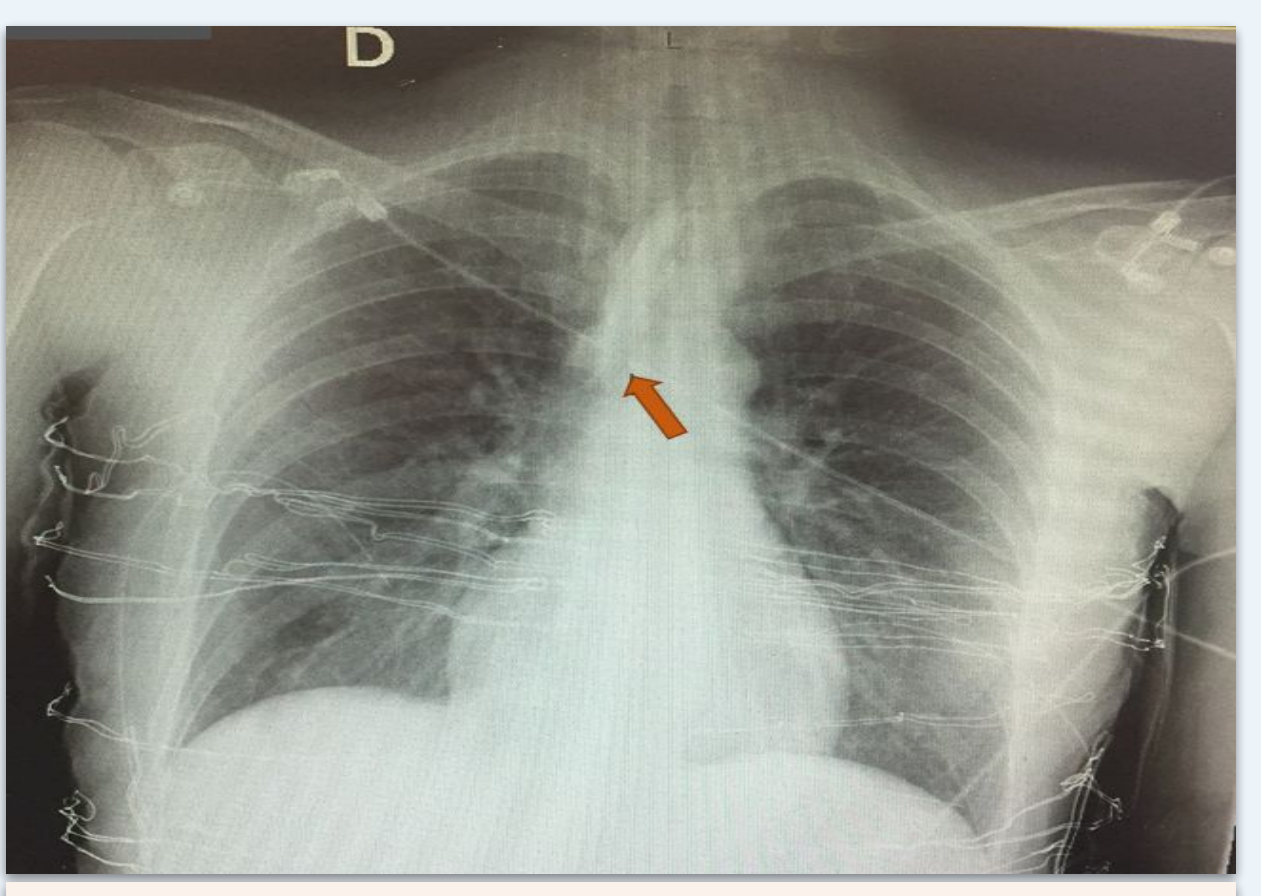

Fig 3. ESP block: Radiographic image with contrast

\section{Conclusions}

- ESP block provides anesthesia at multi-dermatomal levels across posterior, lateral and anterior thoracic wall, making this technique suitable for breast cancer surgery. The main advantage of this block is that it may cover a greater area of the breast and the axilla.

- Continuous infusion ensures optimal postoperative pain control. In conclusion, we present 3 cases of complex breast cancer surgery where continuous ESP block led to an optimal perioperative pain control with a minimum use of opioids and other analgesics.

\section{References}

1. Bonvicini D, Giacomazzi A, Pizzirani E. Use of ultrasound guide of erector spinae plane block in breast surgery. Minerva Anesthesiology 2017; 83(10): IIII-2.

2. Scimia P, Basso Ricci E, Droghetti A, Fusco P. The ultrasound-guided continuous erector spinae plane block for postoperative analgesia in video-assisted thoracoscopic lobectomy. Reg Anesth Pain Med 2017; 42(4): 537. 